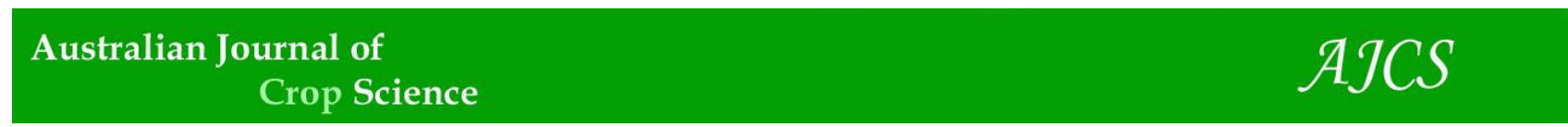

AJCS 12(08):1342-1350 (2018)

ISSN:1835-2707

doi: 10.21475/ajcs.18.12.08.PNE1210

\title{
The within-panicle flowering sequence of hybrid rice affects seed vigour
}

\author{
Aiju Meng, Yan Li, Linmao Zhao, Chunqing Zhang*
}

State Key Laboratory of Crop Biology, College of Agronomy, Shandong Agricultural University, Taian, China, 271018

*Corresponding author: 1039827803@qq.com; cqzhang@sdau.edu.cn

\begin{abstract}
Rice grains located along one panicle do not develop synchronously. Understanding of relationships between upper, middle and basal portions and flowering positions of rice panicles with grain weight, vigour and morphology is important for high-vigour seed production. In this study, four rice $F_{1}$ hybrids were used to analyse grain morphology, 1000-grain weight and seed vigour. The position of the seed on the panicle had a significant influence on seed vigour, with upper seeds having the highest vigour, followed by middle and basal seeds. The flowering sequence within a given branch affected seed size, 1000-grain weight and seed vigour, with differences in 1000-grain weight, mainly as a function of seed length. In each hybrid, the 1000-grain weight and vigour index of primary branches were superior to those of secondary branches. The flowering sequence of the primary grain branch was significantly correlated with 1000-grain weight and seed vigour index, with the highest values recorded at second and third flowering positions. In secondary branches bearing fewer than four grains, 1000-grain weight and seed vigour index exhibited a significant linear relationship with flowering sequence, in which the earliest maturing grains had the highest grain weight and vigour. When secondary branches included more than four grains, grain weight and vigour followed the same parabolic trend as in primary branches. Our findings indicate that 1000-grain weight and seed length can be used as indices of seed vigour.
\end{abstract}

Keywords: 1000-grain weight, flowering sequence, grain morphology, hybrid rice, seed vigour.

\section{Introduction}

Rice (Oryza sativa L.), a globally important food crop, is the basis of food security in many countries. In recent years, especially in developing countries, rice production has not matched the food demand of an increasing population (Chelliah and Gunathila, 2012). To meet this growing demand, rice production has to be raised by at least $70 \%$ over the next three decades (British Council, 2016). The land area devoted to rice cultivation is limited and production cannot be increased by increasing the acreage (British Council, 2016). Therefore, additional applied research is needed to find other ways to increase productivity. Seed quality, one of the most important factors influencing crop quality and yield, is a good indicator of the marketability (Milosevic et al., 2010) and potential performance of seeds in the field. Seed germination and vigour, which are components of seed quality, are thus important characteristics to be considered by farmers before planting (Finch-Savage et al., 2016).

Increasing the grain number per spike is an excellent way to improve rice grain yield potential. A rice spike is composed of spikelets that produce reproductive structures called florets. The grain number and grain weight of rice are influenced by both genetic and environmental factors (Huang Qing, 2014). These two parameters vary unevenly within a spike because of its unbalanced development (Miralles and Slafer, 1995; Chen and Yuan et al., 2007; Ferrante, 2015) and also depend on the spatial position of the grain (Calderini and Reynolds, 2000). In particular, these differences can be attributed to the poor filling of inferior spikelets due to carbon limitations (Murty, 1982; Dreccer et al., 2009; Wang, 1981; Hasegawa, 2017), sink capacity limitations (Kato, 2004), unbalanced hormone levels (Wang, 2015; Yang Zhang, 2006; Zhang Tan et al., 2006), low expression and activity of enzymes involved in sucrose-to-starch conversion (Ishimaru et al., 2005; Jeng et al., 2003; Wang et al., 2008) and impediments to assimilate transportation (Serrago et al., 2013; Yang and Zhang, 2010). Because the ability of developing grains to absorb nutrients differs according to their location on a given panicle, different branch portions and grain positions may cause variation in seed vigour, morphological parameters and grain weight, the latter closely related to grain filling (Fu, 2012). Differences in grain filling rates and final grain weight are mainly due to differences in flowering time (Hato, 1975). Early flowering is associated with strong assimilation ability and promotes rapid grain filling, thereby improving morphological parameters and grain weight. In contrast, late flowering delays grain filling, impedes assimilate acquisition, and frequently leads to inferior grain production and low seed-setting rates (Liu and Xie, 2001; Wang and Cheng, 2004; Yang et al., 1999). Some authors studies the different positions of rice grain weight and seed vigor research (Shi and Zhu, 2002) and also the effect of flowering sequence of spikelets on grain quality of rice. A preliminary analysis showed the effects of seed size and weight on seed vigor of hybrid rice (Liu and Xie, 2001) but the relationship between flowering sequence and seed vigour has not been reported yet.

In this study, we examined variation in grain morphological parameters, grain weight and seed vigour in primary and 
secondary branches and upper, middle and basal portions of panicles of four rice $F_{1}$ hybrids. The aim of our study was to provide a theoretical basis for development of a method for selection of high-vigour rice seed.

\section{Results}

\section{Variation in grain morphological parameters}

Hybrid rice grain morphology (length, width and thickness) and 1000 -grain weight varied according to panicle and grain position in the four $F_{1}$ rice hybrids. Rice grain length and width were highest in upper and middle portions of panicles. Except for grain length in O3S/R1813, differences in grain length, width and thickness were not significant among different panicle positions. In all four $F_{1}$ hybrids, 1000-grain weight followed the trend of upper $>$ middle $>$ basal, with significant differences between upper and basal portions of panicles. Significant differences in 1000-grain weight were also observed between upper and middle portions of panicles of all $F_{1}$ hybrids except for O3S/R1813 (Table 1). The length, width and thicknesses of grains from primary branches were higher than those in secondary branches, but these differences were not significant according to the results of $t$-test (Table 2). Grain lengths were significantly different among different flowering positions of primary and secondary branches, but no significant differences were detected in grain width and thickness for most combinations. Grains at the second and third flowering positions of primary branches were longer than those at fourth, fifth and sixth positions. The order of grain lengths on secondary branches was consistent with the flowering sequence. Grains of O3S/R1813 were significantly longer than those in D8S/R1813, H638S/R1813 and Y58S/R1813. The mean 1000-grain weight per spike of the four hybrid rice varieties was generally higher in primary than secondary branches. In all four hybrids, a parabolic relationship was observed between grain weights of primary branches and flowering sequence, whereas the relationship between grain weights of secondary branches and flowering sequence was linear (Figure 2). The pattern of 1000-grain weight variation was consistent with that of grain length. The grain weight was decreased with flowering sequence. This trend varied slightly among different $F_{1}$ hybrids, with the fastest rate of change measured in the H638S/R1813 hybrid. According to $t$-test, the average 1000 -grain weight of primary vs. secondary branches was not significantly different (Table 2). The highest grain weight was in O3S/R1813, which was consistent with grain length trend.

\section{Vigour index}

Seed vigour index in different panicle regions of the four hybrids $F_{1}$ followed the order of upper $>$ middle $>$ basal (Table 3). No significant difference in germination rate was detected among these three regions. In H638S/R1813 and ShenD8S/R1813 hybridsF1, seed germination potential, germination index and vigour index exhibited significant differences among upper, middle and basal panicle regions.

Except in O3S/R1813 hybrid rice, the seed vigour, germination rate and germination index of primary branch grains were higher than those of secondary branches. According to the $t$-test, vigour-index mean values were not significantly different between primary and secondary branches (Table 4). Significant differences were found in the germination index of primary branch grains at different flowering positions; whereas the same was true for secondary branches. No significant difference was detected in the germination potential and germination rate of most combinations. On primary branches, the germination indexes of seeds formed at second and third flowering position seeds were higher than those of fourth, fifth and sixth positions. On secondary branches, the trend in the seed germination index was consistent with the flowering sequence. Germination indexes of ShenD8S/R1813 and Y58S/R1813 were higher than those of O3S/R1813 and H638S/R1813. Grain numbers of primary and secondary branches differed among $F_{1}$ hybrids (Table 4). The relationship between the seed vigour index of primary branch grains and flowering position followed a parabolic curve (Figure 3). A large difference was observed in the number of grains (Table 4) within secondary branches of four hybrids. In secondary branches bearing three grains, the vigour index was highest at the first flowering position, followed by second and third positions; thus consistent with the flowering sequence. The relationship between seed vigour index and flowering position was linear in H638S/R1813 and Y58S/R1813 and parabolic in ShenD8S/R1813 and O3S/R1813 (Figure 3).

\section{Correlations among different parameters}

A correlation analysis was performed to examine relationships among 1000-grain weight, vigour and flowering position on primary and secondary branches of the four hybrid rice varieties (Table 5). Significant correlations were uncovered between grain weight and flowering position on both primary and secondary branches. This correlation was parabolic in primary branches and linear in secondary ones (Figure 2; Table 5). A parabolic correlation was found between seed vigour and flowering position on primary branches; whereas the same was true for seed vigour and flowering position on secondary branches in ShenD8S/R1813 and O3S/R1813. Seed vigour in H638S/R1813 and Y58S/R1813 was linearly correlated with flowering position on secondary branches (Figure 3; Table 5). Except in the Y58S/R1813 hybrid, grain weight and seed vigour were significantly and positively correlated within upper, middle and basal parts of panicles (Table 5). In each hybrid, 1000 -grain weight and vigour were positively correlated, but no correlation was observed between these two parameters across different $F_{1}$ hybrids. Significant parabolic correlations were detected between both grain weight and seed vigour vs. flowering sequence on primary branches (Table 5). Except for ShenD8S/R1813 and O3S/R1813 the grain weight and seed vigour were linearly correlated with flowering sequence on secondary branches. A parabolic correlation was uncovered between grain length and seed vigour on primary branches at different flowering positions. No correlation was detected between grain length and vigour within upper, middle and basal parts of panicles and among different flowering positions of secondary branches. Except in O3S/R1813, grain length and seed vigour from grains at different flowering positions of primary branches were significantly correlated (Table 5 ). These findings indicate that 1000-grain weight and seed length can be used as indexes of seed vigour. 
Table 1. Morphological parameters of grains from upper, middle and basal parts of panicles.

\begin{tabular}{|c|c|c|c|c|c|}
\hline Hybrid & & $\mathrm{GL}(\mathrm{mm})$ & $\mathrm{GW}(\mathrm{mm})$ & GT $(\mathrm{mm})$ & TGW (g) \\
\hline & Upper & $9.40 a$ & $2.80 a$ & $2.00 \mathrm{a}$ & $24.30 \mathrm{a}$ \\
\hline \multirow[t]{3}{*}{ H638S/R1813 } & Middle & $9.23 a$ & $2.82 a$ & $1.97 a$ & $23.95 b$ \\
\hline & Basal & $9.27 a$ & $2.76 a$ & $1.95 a$ & $23.05 c$ \\
\hline & Upper & $8.53 a$ & $2.64 a$ & $1.80 a$ & $20.41 a$ \\
\hline \multirow[t]{3}{*}{ ShenD8S/R1813 } & Middle & $8.72 a$ & $2.62 a$ & $1.73 a$ & $19.99 b$ \\
\hline & Basal & $8.63 a$ & $2.61 a$ & $1.79 a$ & $19.40 c$ \\
\hline & Upper & $9.10 a$ & $2.51 b$ & $1.77 a$ & $20.70 a$ \\
\hline \multirow[t]{3}{*}{ Y58S/R1813 } & Middle & $9.04 a$ & $2.68 a$ & $1.78 \mathrm{a}$ & $20.31 b$ \\
\hline & Basal & $8.88 a$ & $2.58 b$ & $1.78 a$ & $20.12 b$ \\
\hline & Upper & $9.74 a$ & $2.48 a$ & 1.99a & $24.35 a$ \\
\hline \multirow[t]{2}{*}{ O3S/R1813 } & Middle & $9.60 a b$ & $2.48 a$ & $2.03 a$ & $24.09 a$ \\
\hline & Basal & $9.42 b$ & $2.48 a$ & $1.90 a$ & $23.52 \mathrm{~b}$ \\
\hline
\end{tabular}

In the four rice hybrids $F_{1}$, morphological parameters of grains from upper, middle and basal parts of panicles mean comparison. Means with the same letter are not significantly different according to Fisher's LSD test at P $\leq 0.05$. GL, grain length; GW, grain width; GT, grain thickness; TGW, 1000-grain weight.

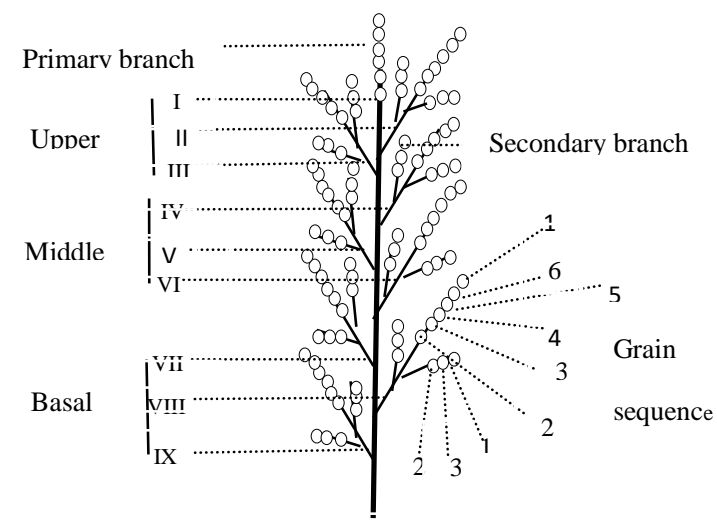

Fig 1. Schematic representation of a rice panicle.

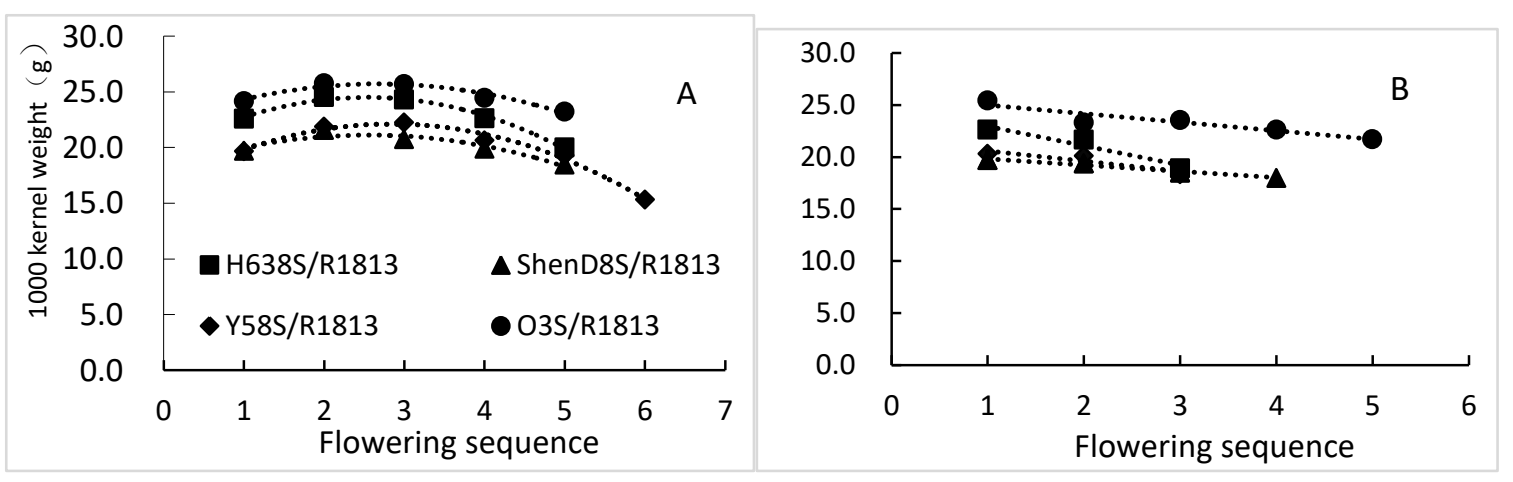

Fig 2. Relationship of 1000-grain weight and flowering sequence in four rice hybrids F1(A-B) .Relationship of 1000-grain weight and flowering sequence on panicle primary branches(A) and secondary branches (B).

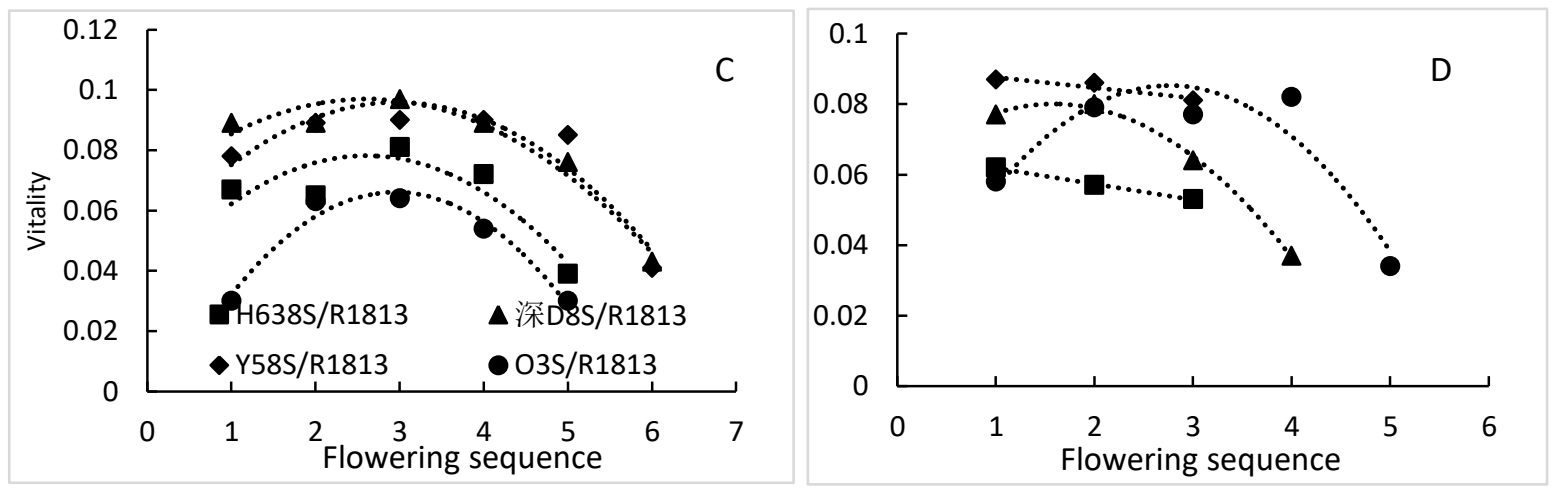

Fig 3. Relationship of seed vigour and flowering sequence in four $F_{1}$ rice hybrids. (C-D) Relationship of seed vigour and flowering sequence on panicle primary branches (C) and second branches (D). 
Table 2. Morphological parameters of grains from different flowering sequence.

\begin{tabular}{|c|c|c|c|c|c|c|}
\hline Hybrid & & & $\mathrm{GL}(\mathrm{mm})$ & $\mathrm{GW}(\mathrm{mm})$ & GT $(\mathrm{mm})$ & TGW (g) \\
\hline \multirow{10}{*}{ H638S/R1813 } & \multirow{6}{*}{$\begin{array}{l}\text { Primary } \\
\text { branch } \\
\text { sequence }\end{array}$} & 1 & $9.62 \mathrm{~cd}$ & $2.82 \mathrm{a}$ & $1.99 a$ & $22.59 \mathrm{~b}$ \\
\hline & & 2 & $9.86 a$ & $2.85 a$ & $2.01 a$ & $24.56 a$ \\
\hline & & 3 & $9.33 a b$ & $2.84 a$ & $2.00 a$ & $24.33 a$ \\
\hline & & 4 & $9.05 b c$ & $2.87 a$ & $1.90 a$ & $22.63 b$ \\
\hline & & 5 & $8.43 d$ & $2.82 a$ & $1.89 a$ & $20.01 \mathrm{c}$ \\
\hline & & $\begin{array}{l}\text { Mean } \\
\text { value }\end{array}$ & 9.15 & 2.85 & 1.94 & 22.82 \\
\hline & \multirow{4}{*}{$\begin{array}{l}\text { Secondary } \\
\text { branch } \\
\text { sequence }\end{array}$} & 1 & $9.42 a$ & $2.84 a$ & $1.89 a$ & $22.63 a$ \\
\hline & & 2 & $9.06 a$ & $2.76 a$ & $1.95 a$ & $21.68 \mathrm{~b}$ \\
\hline & & 3 & $8.34 b$ & $2.85 a$ & $1.94 a$ & $18.93 c$ \\
\hline & & $\begin{array}{l}\text { Mean } \\
\text { value }\end{array}$ & 8.94 & 2.82 & 1.93 & 21.08 \\
\hline \multirow{11}{*}{$\begin{array}{l}\text { ShenD8S/R181 } \\
3\end{array}$} & \multirow{6}{*}{$\begin{array}{l}\text { Primary } \\
\text { branch } \\
\text { sequence }\end{array}$} & 1 & $8.82 \mathrm{~cd}$ & $2.63 a$ & $1.78 a$ & $19.73 c$ \\
\hline & & 2 & $9.22 \mathrm{a}$ & $2.57 a$ & $1.81 a$ & $21.60 a$ \\
\hline & & 3 & $9.10 a b$ & $2.60 a$ & $1.84 a$ & $20.76 b$ \\
\hline & & 4 & $8.63 d$ & $2.66 a$ & $1.74 a$ & $19.89 c$ \\
\hline & & 5 & $8.39 \mathrm{e}$ & $2.72 a$ & $1.73 a$ & $18.47 d$ \\
\hline & & $\begin{array}{l}\text { Mean } \\
\text { value }\end{array}$ & 8.85 & 2.64 & 1.79 & 19.68 \\
\hline & \multirow{5}{*}{$\begin{array}{l}\text { Secondary } \\
\text { branch } \\
\text { sequence }\end{array}$} & 1 & $8.79 a$ & $2.69 a$ & $1.76 \mathrm{~b}$ & $19.72 a$ \\
\hline & & 2 & $8.45 b$ & $2.54 b$ & $1.81 \mathrm{a}$ & $19.39 \mathrm{~b}$ \\
\hline & & 3 & $8.18 c$ & $2.62 a$ & $1.75 b$ & $18.53 c$ \\
\hline & & 4 & $7.84 d$ & $2.51 \mathrm{~b}$ & $1.73 b$ & $18.02 d$ \\
\hline & & $\begin{array}{l}\text { Mean } \\
\text { value }\end{array}$ & 8.32 & 2.59 & 1.76 & 18.92 \\
\hline \multirow{11}{*}{ Y58S/R1813 } & \multirow{8}{*}{$\begin{array}{l}\text { Primary } \\
\text { branch } \\
\text { sequence }\end{array}$} & 1 & $8.90 \mathrm{~d}$ & $2.50 c$ & $1.75 c$ & $19.67 d$ \\
\hline & & 2 & $9.59 a$ & $2.67 a b$ & $1.81 a b$ & $21.88 \mathrm{~b}$ \\
\hline & & 3 & $9.38 b$ & $2.69 a b$ & $1.82 \mathrm{a}$ & $22.26 a$ \\
\hline & & 4 & $9.12 c$ & $2.67 a b$ & $1.81 \mathrm{ab}$ & $20.66 c$ \\
\hline & & 5 & $8.75 \mathrm{e}$ & $2.70 a$ & $1.76 \mathrm{bc}$ & $19.32 \mathrm{e}$ \\
\hline & & 6 & $8.39 f$ & $2.61 b$ & $1.73 c$ & $15.32 f$ \\
\hline & & $\begin{array}{l}\text { Mean } \\
\text { value }\end{array}$ & 9.02 & 2.64 & 1.78 & 20.76 \\
\hline & & 1 & $9.15 a$ & $2.64 a$ & $1.79 a$ & $20.32 a$ \\
\hline & \multirow{3}{*}{$\begin{array}{l}\text { Secondary } \\
\text { branch } \\
\text { sequence }\end{array}$} & 2 & $8.39 b$ & $2.67 a$ & $1.77 a$ & $20.12 a$ \\
\hline & & 3 & $8.39 b$ & $2.67 a$ & $1.77 a$ & $18.38 \mathrm{~b}$ \\
\hline & & $\begin{array}{l}\text { Mean } \\
\text { value }\end{array}$ & 8.64 & 2.66 & 1.78 & 19.61 \\
\hline \multirow{12}{*}{ O3S/R1813 } & \multirow{7}{*}{$\begin{array}{l}\text { Primary } \\
\text { branch } \\
\text { sequence }\end{array}$} & 1 & $9.73 b$ & $2.55 a$ & $1.92 \mathrm{a}$ & $24.17 c$ \\
\hline & & 2 & $10.13 a$ & $2.48 a$ & $1.98 a$ & $25.80 a$ \\
\hline & & 3 & $9.85 a b$ & $2.57 a$ & $1.96 a$ & $25.71 a$ \\
\hline & & 4 & $9.64 b c$ & $2.53 a$ & $1.94 a$ & $24.49 \mathrm{~b}$ \\
\hline & & 5 & $9.40 c$ & $2.56 a$ & $1.93 a$ & $23.22 d$ \\
\hline & & $\begin{array}{l}\text { Mean } \\
\text { value }\end{array}$ & 9.75 & 2.54 & 1.95 & 24.68 \\
\hline & & 1 & $9.92 a$ & $2.48 a$ & $1.96 a$ & $25.46 a$ \\
\hline & \multirow{5}{*}{$\begin{array}{l}\text { Secondary } \\
\text { branch } \\
\text { sequence }\end{array}$} & 2 & $9.56 \mathrm{~b}$ & $2.36 a$ & $1.92 a$ & $23.32 \mathrm{~b}$ \\
\hline & & 3 & $9.18 c$ & $2.44 a$ & $1.93 a$ & $23.58 b$ \\
\hline & & 4 & $9.21 \mathrm{c}$ & $2.43 a$ & $1.96 a$ & $22.65 c$ \\
\hline & & 5 & $8.88 d$ & $2.47 a$ & $1.82 \mathrm{a}$ & $21.29 d$ \\
\hline & & $\begin{array}{l}\text { Mean } \\
\text { value }\end{array}$ & 9.35 & 2.43 & 1.92 & 23.26 \\
\hline
\end{tabular}

In four $F_{1}$ rice hybrids, morphological parameters of grains from different flowering sequence. The mean value of the two stems. Means with the same letter are not significantly different according to Fisher's LSD test at P $\leq 0.05$. GL, grain length; GW, grain width; GT, grain thickness; TGW, 1000-grain weight. 
Table 3. Seed vigour index of grains for the upper, middle and basal parts of panicles.

\begin{tabular}{|c|c|c|c|c|c|}
\hline Hybrid & & GR (\%) & GP (\%) & $\mathrm{GI}$ & $\mathrm{VI}$ \\
\hline & Upper & $63 a$ & $39 a$ & $5.86 a$ & $0.063 a$ \\
\hline \multirow[t]{3}{*}{ H638S/R1813 } & Middle & $64 a$ & $40 a$ & $5.96 a$ & $0.061 a$ \\
\hline & Basal & $61 a$ & $30 b$ & $3.76 b$ & $0.033 b$ \\
\hline & Upper & $97 a$ & $60 a$ & $8.95 a$ & $0.071 a$ \\
\hline \multirow[t]{3}{*}{ ShenD8S/R1813 } & Middle & $92 a$ & $57 a b$ & $8.53 a b$ & $0.067 a b$ \\
\hline & Basal & $82 a$ & $46 b$ & $7.45 b$ & $0.060 \mathrm{~b}$ \\
\hline & Upper & $97 a$ & $92 a$ & $11.53 a$ & $0.087 a$ \\
\hline \multirow[t]{3}{*}{ Y58S/R1813 } & Middle & $95 a$ & $93 a$ & 11.27ab & $0.084 a$ \\
\hline & Basal & $95 a$ & $88 a$ & $11.06 b$ & $0.079 a$ \\
\hline & Upper & $96 a$ & $89 a$ & $10.41 a$ & $0.097 a$ \\
\hline \multirow[t]{2}{*}{ O3S/R1813 } & Middle & $89 a$ & $79 a$ & $9.78 a$ & $0.087 a$ \\
\hline & Basal & $87 a$ & $81 a$ & $9.76 a$ & $0.073 a$ \\
\hline
\end{tabular}

In four $F_{1}$ rice hybrids, seed vigour index of grains from upper, middle and basal parts of panicles mean comparison. Means with the same letter are not significantly different according to Fisher's LSD test at $\mathrm{P} \leq 0.05$. GR, germination rate; GP, germination potential; GI, germination index; VI, vigour index.

Table 4. Seed vigour index of grains from different flowering sequence.

\begin{tabular}{|c|c|c|c|c|c|c|}
\hline Hybrid & & & GR (\%) & GP $(\%)$ & GI & $\mathrm{VI}$ \\
\hline & & 1 & $69 a$ & $57 a b$ & $7.5 a$ & $0.067 a$ \\
\hline & & 2 & $63 a b$ & $50 \mathrm{bc}$ & $7.1 \mathrm{a}$ & $0.065 a$ \\
\hline & Primary branch sequence & 3 & $62 \mathrm{ab}$ & $60 a$ & $7.5 a$ & $0.081 a$ \\
\hline & & 4 & $63 a b$ & $53 a b c$ & $7.1 \mathrm{a}$ & $0.072 \mathrm{a}$ \\
\hline & & 5 & $54 b$ & $48 c$ & $5.0 \mathrm{~b}$ & $0.040 \mathrm{~b}$ \\
\hline & & Mean value & 62 & 54 & 6.8 & 0.065 \\
\hline \multirow[t]{12}{*}{ H638S/R1813 } & & 1 & $60 a$ & $49 a$ & $6.6 a$ & $0.062 a$ \\
\hline & Secondary branch sequence & 2 & $57 a$ & $45 a$ & $6.2 \mathrm{a}$ & $0.057 a$ \\
\hline & & 3 & $58 a$ & $54 a$ & $5.4 a$ & $0.053 a$ \\
\hline & & Mean value & 58 & 49 & 6.1 & 0.057 \\
\hline & T value & & 1.515 & 1.251 & 1.318 & 1.048 \\
\hline & & 1 & $95 a$ & $73 a$ & $10.2 a$ & $0.089 a$ \\
\hline & & 2 & $93 a$ & $77 a$ & $10.1 a$ & $0.089 a$ \\
\hline & Primary branch sequence & 3 & $97 a$ & $79 a$ & $10.5 a$ & $0.097 a$ \\
\hline & & 4 & $95 a$ & $72 a$ & $10.1 \mathrm{a}$ & $0.089 a$ \\
\hline & & 5 & $91 a$ & $69 a$ & $9.7 a$ & $0.076 a$ \\
\hline & & 6 & $94 a$ & $72 a$ & $6.0 b$ & $0.043 b$ \\
\hline & & Mean value & 94 & 74 & 9.4 & 0.081 \\
\hline \multirow[t]{13}{*}{ ShenD8S/R1813 } & & 1 & $90 a b$ & $63 a$ & $9.4 a$ & $0.077 a$ \\
\hline & Secondary branch sequence & 2 & $89 a b$ & $63 a$ & $9.4 a$ & $0.080 \mathrm{a}$ \\
\hline & & 3 & $95 a$ & $59 a$ & $9.5 a$ & $0.089 a$ \\
\hline & & 4 & $84 b$ & $61 a$ & $5.1 b$ & $0.037 a$ \\
\hline & & Mean value & 90 & 62 & 8.4 & 0.071 \\
\hline & T value & & 1.941 & 6.843 & 0.842 & 0.695 \\
\hline & & 1 & $95 a$ & $72 a$ & $10.1 a$ & $0.078 \mathrm{~b}$ \\
\hline & & 2 & $95 a$ & $77 a$ & $10.5 a$ & $0.089 a b$ \\
\hline & Primary branch sequence & 3 & $95 a$ & $82 a$ & $10.6 a$ & $0.09 a$ \\
\hline & & 4 & $91 a$ & $81 a$ & $10.4 a$ & $0.09 a$ \\
\hline & & 5 & $95 a$ & $85 a$ & $10.8 a$ & $0.085 a b$ \\
\hline & & 6 & $95 a$ & $86 a$ & $6.1 b$ & $0.041 c$ \\
\hline & & Mean value & 94 & 81 & 9.8 & 0.079 \\
\hline \multirow[t]{10}{*}{ Y58S/R1813 } & & 1 & $96 a$ & $85 a$ & $10.8 a$ & $0.086 a$ \\
\hline & Secondary & 2 & $93 a$ & $83 a$ & $10.9 a$ & $0.089 a$ \\
\hline & sequence & 3 & $94 a$ & $81 a$ & $10.7 a$ & $0.084 a$ \\
\hline & & Mean value & 94 & 83 & 10.8 & 0.086 \\
\hline & T value & & 0 & -1.028 & -1.422 & -0.946 \\
\hline & & 1 & $93 a b$ & $31 a$ & $4.5 c$ & $0.030 c$ \\
\hline & Primary branch sequence & 2 & $90 b$ & $49 a$ & $8.4 a$ & $0.063 a$ \\
\hline & & 3 & $93 a b$ & $43 a$ & $8.5 a$ & $0.064 a$ \\
\hline & & 4 & $91 \mathrm{ab}$ & $53 a$ & $6.9 b$ & $0.054 b$ \\
\hline & & 5 & $96 a$ & $41 a$ & $4.3 c$ & $0.030 c$ \\
\hline \multirow[t]{8}{*}{ O3S/R1813 } & & Mean value & 93 & 43 & 6.5 & 0.048 \\
\hline & & 1 & $93 a$ & $40 \mathrm{~b}$ & $8.4 a$ & $0.058 b$ \\
\hline & & 2 & $89 a$ & $69 a$ & $9.5 a$ & $0.079 a$ \\
\hline & Secondary branch sequence & 3 & $93 a$ & $67 a$ & $9.4 a$ & $0.077 a$ \\
\hline & & 4 & $89 a$ & $71 a$ & $9.5 a$ & $0.082 a$ \\
\hline & & 5 & $87 a$ & $61 a$ & $4.9 b$ & $0.034 c$ \\
\hline & & Mean value & 90 & 62 & 8.3 & 0.066 \\
\hline & T value & & 1.518 & -2.68 & -1.433 & -1.505 \\
\hline
\end{tabular}

In four rice hybrids $F_{1}$, Seed vigour index of grains from different flowering sequence mean comparison. Means with the same letter are not significantly different according to Fisher's LSD test at $\mathrm{P}=0.05$. GR, germination rate; GP, germination potential; GI, germination index; VI, vigour index. Different lowercase letters in the same row indicate significant differences between one or more sequences $\left(t_{6,0.025}=2.447 ; t_{8,0.025}=2.306\right)$. 
Table 5. Results of correlation analyses of different rice grain parameters.

\begin{tabular}{|c|c|c|c|c|c|c|c|c|}
\hline \multirow{2}{*}{ Treatment } & \multicolumn{2}{|l|}{ H638S/R1813 } & \multicolumn{2}{|c|}{ ShenD8S/R1813 } & \multicolumn{2}{|c|}{ Y58S/R1813 } & \multicolumn{2}{|c|}{ O3S/R1813 } \\
\hline & Equation & Coefficient(r) & Equation & Coefficient(r) & Equation & Coefficient( $r$ ) & Equation & Coefficient( $r$ ) \\
\hline A & $\begin{array}{c}y=-0.7602 x^{2}+3.8518 x+19 \\
633\end{array}$ & $0.994 * *$ & $\begin{array}{c}y=-0.2834 x^{2}+1.38 \\
99 x+19.114\end{array}$ & $0.932^{*}$ & $\begin{array}{c}y=-0.6497 x^{2}+3.7064 x+ \\
16.784\end{array}$ & $0.961^{* *}$ & $\begin{array}{c}y=-0.4936 x^{2}+2.64 \\
1 x+22.185\end{array}$ & $0.970^{* *}$ \\
\hline B & $y=-1.852 x+24.786$ & $0.963^{*}$ & $y=-0.596 x+20.406$ & $0.968^{* *}$ & $y=-0.978 x+21.551$ & 0.91 & $\begin{array}{c}y=-0.8156 x+25.79 \\
1\end{array}$ & $0.932^{* *}$ \\
\hline C & $\begin{array}{c}y=-0.0064 x^{2}+0.0336 x+0.0 \\
244\end{array}$ & $0.890^{*}$ & $\begin{array}{c}y=-0.0044 x^{2}+0.02 \\
32 x+0.0667\end{array}$ & $0.979^{* *}$ & $\begin{array}{c}y=-0.0053 x^{2}+0.0317 x+ \\
0.0487\end{array}$ & $0.942^{* *}$ & $\begin{array}{c}y=-0.0089 x^{2}+0.05 \\
27 x-0.0116\end{array}$ & $0.982^{* *}$ \\
\hline D & $y=-0.012 x+0.0763$ & $0.948^{*}$ & $\begin{array}{c}y=-0.0136 x+0.098 \\
5\end{array}$ & $-0.896 *$ & $y=-0.0015 x+0.0883$ & $0.982^{*}$ & $\begin{array}{c}y=-0.0089 x^{2}+0.05 \\
27 x-0.0116\end{array}$ & $0.982^{* *}$ \\
\hline E & $y=0.0254 x-0.5501$ & $0.966^{*}$ & $y=0.0098 x-0.1301$ & $0.995^{* *}$ & $y=0.0127 x-0.1755$ & 0.942 & $y=0.0282 x-0.5501$ & $0.993^{* *}$ \\
\hline $\mathrm{F}$ & $\begin{array}{c}y=-0.0024 x^{2}+0.1143 x-1.2 \\
847\end{array}$ & $0.928^{*}$ & $\begin{array}{c}y=-0.0057 x^{2}+0.23 \\
5 x-2.3211\end{array}$ & $0.983^{*}$ & $\begin{array}{c}y=-0.0125 x^{2}+0.5321 x- \\
5.5634\end{array}$ & $0.918^{*}$ & $\begin{array}{c}y=-0.001 x^{2}-0.034 \\
4 x+0.293\end{array}$ & $0.910^{*}$ \\
\hline G & $y=0.0022 x+0.0107$ & 0.944 & $\begin{aligned} y= & -0.0507 x^{2}+1.93 \\
& 21 x-18.296\end{aligned}$ & 0.903 & $y=0.003 x+0.0258$ & $0.998^{* *}$ & $\begin{array}{c}y=-0.0103 x^{2}+0.49 \\
09 x-5.7635\end{array}$ & $0.932^{*}$ \\
\hline $\mathrm{H}$ & $y=-0.0741 x+0.796$ & $0.986^{*}$ & $\begin{array}{c}y=-0.0277 x+0.304 \\
7\end{array}$ & 0.526 & $y=0.0353 x-0.2347$ & $0.994^{* *}$ & $y=0.0751 x-0.6346$ & $0.999 * *$ \\
\hline 1 & $\begin{array}{c}y=-0.0464 x^{2}+0.8638 x-3.9 \\
473\end{array}$ & $0.971^{* *}$ & $\begin{array}{c}y=-0.0487 x^{2}+0.87 \\
56 x-3.8424\end{array}$ & $0.925^{*}$ & $\begin{array}{c}y=-0.1539 x^{2}+2.8701 x- \\
13.281\end{array}$ & $0.956^{*}$ & $\begin{array}{c}y=-0.0216 x^{2}+0.46 \\
74 x-2.4572\end{array}$ & 0.730 \\
\hline J & $y=0.0079 x+0.0136$ & $0.978^{* *}$ & $\begin{array}{c}y=-0.1264 x^{2}+2.13 \\
55 x-8.9326\end{array}$ & 0.0897 & $y=0.0046 x+0.0449$ & 0.629 & $\begin{aligned} y= & -0.1479 x^{2}+2.79 \\
& 89 x-13.157\end{aligned}$ & $0.976^{* *}$ \\
\hline K & $y=2.8028 x-3.1227$ & $0.852^{*}$ & $y=3.3252 x-9.2772$ & $0.950^{* *}$ & $y=3.5986 x-12.163$ & $0.946 * *$ & $y=3.6891 x-11.29$ & $0.914^{*}$ \\
\hline $\mathrm{L}$ & $y=3.4857 \times 10.08$ & $0.997^{*}$ & $y=1.9123 x+3.0097$ & $0.972 * *$ & $y=1.4164 x+7.3625$ & 0.582 & $y=3.1678 x-6.2746$ & 0.915 \\
\hline
\end{tabular}

(A) 1000-grain weight vs. flowering sequence on primary branches; (B) 1000-grain weight vs. flowering sequence on secondary branches; (C) Seed vigour vs. flowering sequence on primary branches; (D) Seed vigour vs. flowering sequence on secondary branches; (E) Seed vigour vs. 1000-grain weight from upper, middle and basal parts of panicles; (F) Seed vigour vs. 1000-grain weight at different flowering sequences of primary branches; (G) Seed vigour vs. 1000-grain weight on secondary branches; (H) Seed vigour vs. grain length from upper, middle and basal parts of panicles; (I) Seed vigour vs. grain length from different flowering sequences of primary branches; (J) Seed vigour vs. grain length at different flowering sequences of secondary branches; (K) 1000-grain weight vs. grain length at different flowering sequences of primary branches; (L) 1000-grain weight vs. grain length at different flowering sequences of secondary branches.* and $* *$ Level of significance at $p<0.05$ and $p<0.01$ respectively. $r_{0.05}, 2=0.950 ; r_{0.01}, 2=0.990 ; r_{0.05}, 4=0.811 ; r_{0.01}, 4=0.917$

\section{Discussion}

The potential ability of a rice hybrid to exhibit increased yield, grain vigour and grain weight is affected by genetic and environmental factors (Cheng et al., 2007). Flowering position has a significant effect on seed vigour. The duration and sequence of grain filling in rice leads to different levels of grain plumpness in panicles. For example, rice grains from upper and middle portions are characterized by higher seed vigour than basal grains, and seed vigour is higher on primary than secondary branches. Grain filling in rice is a complex physiological process that is subject to significant geneenvironment interactive effects (Lin et al., 2011). The quality of developing grains is also shaped by temporal and spatial differences in gene expression and regulation (Lin et al., 2011). A morphological analysis found that lower, inferior rice grains experienced a period of stagnation prior to the start of grain filling after fertilization (Ishimaru et al., 2003), whereas superior grains immediately entered a period of rapid accumulation of starch. Superior grains, which are located in the upper and middle parts of panicles, generally fill early and develop quickly (Lin et al., 2011). Unsynchronized grain filling is frequently observed in large-panicle-type and conventional hybrid rice varieties (Lin et al., 2011). Many studies have shown that the low 1000 -grain weight and seed setting rate of inferior grains limit the high yield potential of rice. This result is mainly due to the asynchrony of grain filling between superior and inferior grains (Yang, 2010; Lin et al., 2011; Ishimaru et al., 2003, 2005; Huang and Liang, 2003; Huang, 2005). Grain weight can differ significantly among grain positions, not only in rice but also in crops such as wheat, maize and rapeseed (Gonzalez, 1993; Gao et al., 2001; Li and Shi, 2001; Shi and Zhu, 2002; Li, 2007). Within panicles, grain morphological parameters, grain weight and vigour were lowest at the base, with significant differences in seed vigour uncovered among lower, middle and upper parts of panicles. We uncovered a significant correlation between grain weight and vigour in different parts of the panicle, a result inconsistent with the observations of Lin et al., (2014). In terms of specific flowering positions, we found that grain morphological parameters, grain weight and seed vigour were higher in grains on primary than secondary branches, but these differences were not significant according to a $t$-test. The 1000-grain weight and vigour of grains from primary branches was parabolically correlated with flowering sequence. The shape of these relationship curves differed among the four $F_{1}$ rice hybrids, possibly because of differences in their grain-filling rates. Within primary branches, the seed germination index was highest at second and third 
flowering positions, followed by fourth, fifth and sixth positions. These results indicate that flowering sequence has a strong influence on seed vigour. The observed trends in 1000-grain weight in upper, middle and basal panicle portions and grain lengths of primary and secondary branches indicate that these two parameters can be used for seed grading. There is wide variation in significance of difference among traits of hybrids, in which not all the hybrids show similar significance results. This might be due to the genetic makeup differed between them.

In wheat, grain number and grain weight have been reported to differ greatly between spikelets according to their positions on the spike (Pan, et al., 2005; Pei, et al., 2008; HuiJuan, et al,. 2009). This positional variation also occurs in rice. For example, large differences have been observed between rice florets in terms of vascular bundle sizes and quantities, sink capacity, endogenous hormone levels, carbon amounts, activity and/or expression of enzymes involved in sucrose-to-starch conversion, and assimilate transportation, resulting in asynchronous grain development and nutrient accumulation as well as yield differences among spikelets and grains (Murty and Murty, 1982; Dreccer et al., 2009; Wang, 1981; Kato, 2004; Zhang and Tan et al., 2006; Ishimaru et al, 2005; Jeng et al, 2003; Wang et al, 2008). In rice, water loss in grains at the first flowering position of primary branches was more pronounced and occurred earlier than other grains, both before and after grain filling, which may partly be accounted for the low grain weight at the first grain position (Dong, 2011; Xue, 2014). Seed vigour is significantly correlated with the first flowering position of primary branches 1000 -grain weight, which is one reason why seed vigour at the first flowering position was not the highest. The flowering sequence in rice has a significant effect on seed vigour. Within the same branch, the flowering sequence affects seed vigour, with differences in 1000-grain weight, mainly reflecting differences in grain length. Screening for high-vigour seeds on upper and middle parts of panicles can be based on 1000-grain weight. Because grain length and seed vigour at different flowering positions of primary branches are significantly correlated, low-vigour seeds on the upper and middle parts can be filtered out using a nest sieve. These results provide a theoretical basis for the screening of high-vigour seeds.

\section{Materials and Methods}

\section{Seed material}

We used indica rice sterile lines H638S, ShenD8S, Y58S and O3S as female parent, all crossed with the same male parent (R1813) to produce $F_{1}$ hybrids as experimental materials. Seeds of these materials were initially provided by the Yuan Longping Agricultural High Tech Co. (China).

\section{Panicle morphology}

Plants of the four sterile lines differed in terms of panicle branching patterns. Panicles of ShenD8S/R1813 and Y58S/R1813 were generally composed of 9 to 10 primary branches, with upper and basal portions each consisting of 3 primary branches and the middle portion comprising 3 to 4 . In H638S/R1813 and O3S/R1813, panicles generally consisted of 10 to 11 primary branches; the upper and basal portions each included 3 to 4 primary branches, and the middle portion was composed of 4 . To compare flowering sequence and grain characteristics, we labelled the five or six topmost flowering positions on primary branches and the three to four topmost positions on secondary branches according to the order of flowering as indicated in Figure1.

\section{Measurements of morphological parameters and grain weight}

Dry grains were collected from different flowering positions of primary and secondary branches and stored in small beakers labelled with the corresponding position until harvesting was completed (Figure 1). In this study, grain moisture content was measured using a near-infrared grain analyser, while grain length and width were measured automatically with a scanner. The thickness of 10 grains from each sample group was measured with a vernier caliper. The seeds were weighed on a F50-11-type electronic balance. After counting the number of grains, 1000-grain weight was then calculated.

\section{Seedling growth test}

Seedling growth tests were conducted according to the GB3543-1995 standard (Chinese,1995). After sowing seeds on sand at $25^{\circ} \mathrm{C}$ in a seed germination chamber, the number of germinating seedlings was recorded daily for 10 days. Lengths of seedlings (portions above root nodes) and maximum root lengths were recorded. After heating in an envelope in an oven (Shanghai Science Instrument Co., Shanghai, China) for half an hour at $105^{\circ} \mathrm{C}$, the seedlings and roots were kept at $80^{\circ} \mathrm{C}$ until constant weight. Seed germination potential was calculated as the number of seeds germinating normally within the first 4 days/number of seeds tested for germination $\times 100$. Germination rate was calculated as the number of seeds germinating normally within the first 4 days/number of seeds tested for germination $\times 100$. Germination index $(G I)$ was calculated according to the formula $\mathrm{Gl}=\Sigma \mathrm{Gt} / \mathrm{At}$, where $\mathrm{Gt}$ is the number of seeds germinated after 10 days and At is the number of days (Zhang and Wang 2006). Vigour index (VI) was calculated according to the formula $\mathrm{VI}=\mathrm{GI} \times \mathrm{S}$, where $\mathrm{S}$ is seedling dry weight (Zhang and Wang 2006).

\section{Experimental design and statistical analysis}

All statistical analyses were conducted in IBM SPSS version 19. Data were analysed by one-way ANOVA (complete randomised design). Fisher's least significant difference test $(P \leq 0.05)$ was used to detect significant differences between treatment means. A one-sample $t$-test was used as indicated.

\section{Conclusion}

The flowering sequence in rice has a significant effect on seed vigour. Within the same branch, the flowering sequence affects grain morphological parameters, 1000-grain weight and seed vigour, with differences in 1000-grain weight mainly reflecting differences in grain length. Screening for high-vigour seeds on upper and middle parts of panicles can be based on 1000 -grain weight. Because grain length and seed vigour at different flowering positions of primary branches are significantly correlated, low-vigour seeds on the upper and middle parts can be filtered out using a nest sieve. These results provide a theoretical basis for the screening of 
igh-vigour seeds.

\section{Acknowledgements}

This study was financially supported by a Commonweal Industry (Agriculture) Science and Technology Project grant (201303005).

\section{References}

British Council (2016) Necesitamos arroz para alimentar al mundo.

Calderini DF, Reynolds MP (2000) Changes in grain weight as a consequence of de-graining treatments at pre- and post-anthesis in synthetic hexaploid lines of wheat. Aust J Plant Physiol. 27:183-191.

Chelliah S, Gunathilagaraj K (2012) Pest management in rice-current status and future prospects. Rice Knowledge Management Portal (RKMP) Directorate of Rice Research, Rajendranagar, Hyderabad, India. http://www.rkmp.co.in/

Chen Y, Yuan L, Wang X, Zhang D, Chen J, Deng Q, et al (2007) Relationship between grain yield and leaf photosynthetic rate in super hybrid rice. J Plant Physiol Mole Biol. 33: 235-243.

Chen Y, Yuan LP, Wang XH, Zhang DY, Chen J, Deng QY, et al (2007) Relationship between grain yield and leaf photosynthetic rate in super hybrid rice.J Plant Physiol Moler Biol. 33: 235.

Chunqing Zhang, Jianhua Wang (2006) Seed testing. Seed germination test, 4rd edn. Chinese.

Dreccer MF, van Herwaarden AF, Chapman SC (2009) Grain number and grain weight in wheat lines contrasting for stem water soluble carbohydrate concentration. Field Crops Res. 112: 43-54.

Dong M, Xie Y, Qiao Z et al (2011) Dynamic accumulation of starch and protein in different grain positions of rice. Chinese J Rice Science. 25: 297-306.

Ferrante A, Savin R, Slafer GA (2015) Relationship between fruiting efficiency and grain weight in durum wheat. Field Crops Res. 177: 109-116.

Finch-Savage WE, Bassel GW (2016) Seed vigour and crop establishment: extending performance beyond adaptation. J Exp Bot. 67(3):567.

Fu J, Xu Y Cheng L et al. (2012) Post-anthesis changes in activities of enzymes related to starch synthesis and contents of hormones in superior and inferior spikeletsand their relation with grain filling of super rice. Chinese J Rice Sci. 26: 302-310.

Gonzalez E (1993) Effect of seed size on germination and seedling vigor of virola-koschnyi barb. Forest Ecology Management. 57: 275-281.

Gao H, Zou L, Xu Y (2001) Study on the relationship between seed weight and germination of soybean and maize seed. Seed. 21: 68-70.

Hasegawa T, Tao L, Yin X, Yan Z, Boote K, Baker J (2017) Science report .Causes of variation among rice models in yield response to $\mathrm{co} 2$ examined with free-air co2 enrichment and growth chamber experiments, 7rd edn.

Hato $Y$ (1975) Rice grain dry matter increasing process. Annals Crop Soc Jap. 44: 431-437.

Huang J, Liang K (2003) Changes of endogeous hormone contents during grain development in different types of rice. Chinese J Eco-Agric. 11:11-13.
Huang S, Zou Y Liu C (2005) Setting physiology of the superior and inferior grains of hybrid rice liang you pei-jiu. Crop J. 31:102-107.

HuiJuan Q, JinCai L, XueShan S, FengZhen W, ChengYu W, ShengJun Z (2009) Effects of plant density and seeding date on accumulation and translocation of dry matter and nitrogen in winter wheat cultivar Lankao Aizao 8. Acta Agron Sin. 35: 124-131.

Huang Q (2014) The study on the formation rule of rice seed with high seed vigor and the optimal harvest time. Full time master's degree thesis of Hunan Agricultural University.

Ishimaru T, Hirose T, Matsuda T, Goto A, Takahashi K, Sasaki H (2005) Expression patterns of genes encoding carbohydrate-metabolizing enzymes and their relationship to grain filling in rice (Oryza sativa L.): comparison of caryopses located at different positions in a panicle. Plant Cell Physiol. 46: 620-628.

Ishimaru T, Matsuda T, Ohsugi R, Yamagishi T (2003) Morphological development of rice caryopses located at the different positions in a panicle from early to middle stage of grain filling. Funct Plant Biol. 30: 1139-1149.

Jeng T, Wang C, Chen C, Sung J (2003) Effects of grain position on the panicle on starch biosynthetic enzyme activity in developing grains of rice cultivar Tainung 67 and its NaN3-induced mutant. J Agric Sci. 141: 303-311.

Kato T (2004) Effect of spikelet removal on the grain filling of Akenohoshi, a rice cultivar with numerous spikelets in a panicle. J Agric Sci. 142: 177-181.

Liu L, Xie Gi (2001) Effect of flowering sequence of spikelets on endosperm cells and grain quality of rice. Chinese J Rice Sci.15: 119-124.

Lin W, Li Z, Zhang Z (2011) Developmental genetic and molecular ecological properties of rice grain filling. Chinese $J$ Eco-Agric. 19: 1237-1242.

Li M (2007) Study on the difference of grain weight and seed vigor among the different parts of the rape. Chinese Agric Sci Bull Agron. 284-287.

Li M, Shi X (2001) Study on the difference of grain weight and seed vigor among the different parts of the wheat. Chinese Agric Sci Bull. 17: 14-16.

Milosevic M, Vujakovic M, Karagic D (2010) Vigour tests as indicators of seed viability. OAlib J. 42:103-118.

Miralles DJ, Slafer GA (1995) Individual grain weight responses to genetic reduction in culm length in wheat as affected by source-sink manipulations. Field Crops Res. 43: 55-66.

Murty P, Murty K (1982) Spikelet sterility in relation to nitrogen and carbohydrate contents in rice. Indian J Plant Physiol. 25: 40-48.

Pan J, Jiang D, Cao WX, Sun CF (2005) Effects of spikelet and grain positions on grain number, weight and protein content of wheat spike. Acta Agron Sinica. 31: 431-437.

Pei XX, Wang JA, Dang JY, Wang XB, Zhang DY (2008) Effects of spikelet and grain position on fertile spikelet number, grain weight and quality of wheat. Scientia Agric Sinica. 41: 381-390.

Serrago RA, Alzueta I, Savin R, Slafer GA (2013) Understanding grain yield responses to source-sink ratios during grain filling in wheat and barley under contrasting environments. Field Crops Res. 150: 42-51.

Shi X, Zhu C (2002) The different positions of rice grain weight and seed vigor research. Seed. 38-40. 
Wang E, Wang J, Zhu X, Hao W, Wang L, Li Q (2008) Control of rice grain-filling and yield by a gene with a potential signature of domestication. Nat Genet. 40: 1370-1374.

Wang F, Cheng Y (2004) The physiological mechanism of rice grain quality difference in grain filling process was discussed. Seed. 23: 31-35.

Wang Y (1981) Effectiveness of supplied nitrogen at the primordial panicle stage on rice characteristics and yields. Int Rice Res Newsletter. 6: 23-24.

Wang Z, Xu Y, Chen T, Zhang H, Yang J, Zhang J (2015) Abscisic acid and the key enzymes and genes in sucrose-to-starch conversion in rice spikelets in response to soil drying during grain filling. Planta. 241: 1091-1107.
Xue T (2014) Preliminary study on the relationship between morphological, physical characteristics and seed vigor in indica hybrid rice. Full time master's degree thesis of Zhejiang agriculture and Forestry University.

Yang J, Liu L, Wang Z (1999) Rice glume flowering time of endosperm development and its physiological mechanism. Scientia Agric Sinica. 32: 44-517.

Yang J, Zhang J (2006) Grain filling of cereals under soil drying. New Phytol. 169: 223-236.

Yang JC, Zhang JH (2010) Crop management techniques to enhance harvest index in rice. Journal of Experamental Botany. 61: 3177-3189.

Yang J (2010) Mechanism and regulation of grain filling in Rice. Crop J. 36: 2011-2019. 